\title{
Effect of Axial Resolution on PET image data: 2D vs. 3D
}

\author{
TR Oakes ${ }^{1}$, JE Holden ${ }^{2,3}$, RW Pyzalski ${ }^{3}$, AD Roberts ${ }^{1,4}$, \\ WD Brown ${ }^{3,2}$, RJ Nickles ${ }^{2}$, RJ Davidson ${ }^{1,4}$ \\ University of Wisconsin-Madison,
} Depts. of Psychology ${ }^{1}$, Medical Physics ${ }^{2}$, Radiology ${ }^{3}$, Psychiatry ${ }^{4}$
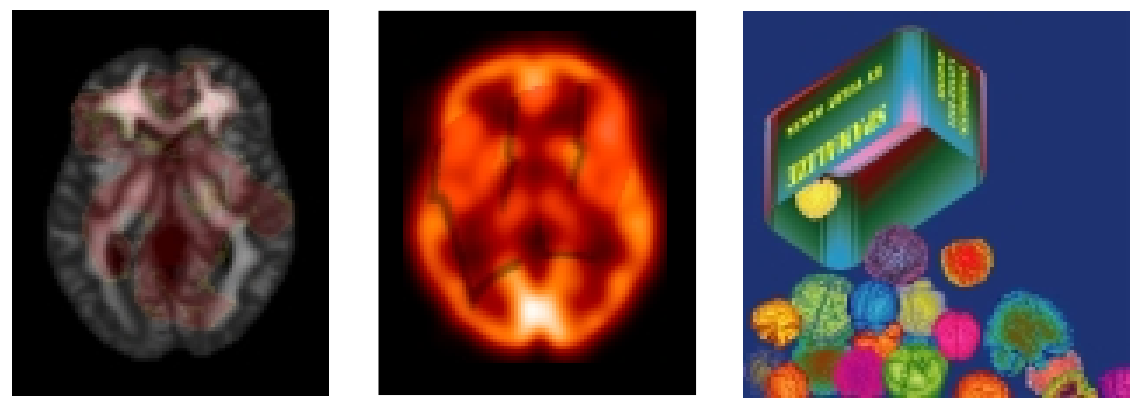

\section{Abstract:}

We compared 12 pairs of cerebral $\left[{ }^{18} \mathrm{~F}\right]$-fluoro-deoxyglucose (FDG) 2D/3D image sets from a GE/Advance PET scanner, incorporating the actual corrections used on human subjects. FDG images were acquired as: 2D scan 50-80 min post-injection (PI); transmission scan 82-92 min PI, 3D scan 95-105 min PI. The emission countrate was $<5 \%$ of the transmission countrate for the $\mathrm{T}+\mathrm{E}$ correction. Data were corrected with vendor-supplied software (normalization, attenuation, scatter, calibration) and reconstructed using similar Hanning filters (2D:4.0, 3D radial:4.0, 3D axial:8.5). The $3 \mathrm{D}$ data had $\sim$ twice the coincident counts as the $2 \mathrm{D}$ data prior to corrections. Previously published values for spatial axial resolution in 2D (DeGrado 1994) and 3D (Lewellen 1996) modes were used to model the differential axial smoothing at each image voxel. This model was then applied to the 2D FDG images as well as to a spherical phantom image; the resulting smoothed data indicate the published difference in axial resolution between 2D and 3D modes can account for 30-40\% of the differences between these image sets.

A phantom containing spherical hot- and cool-spots in a warm background was scanned for a variety of time-fame durations $(30,15,5,1 \mathrm{~min})$ to mimic a typical human cerebral FDG PET scan. Only for the 1-minute frame is there a clear advantage to using 3D mode; for the other longer frames which are more typical of a human FDG scan protocol, the increased accuracy of 2D mode outweighs any small gain in counting statistics. 


\section{Why?}

- There are obvious visual differences between images acquired in 2D and 3D modes.

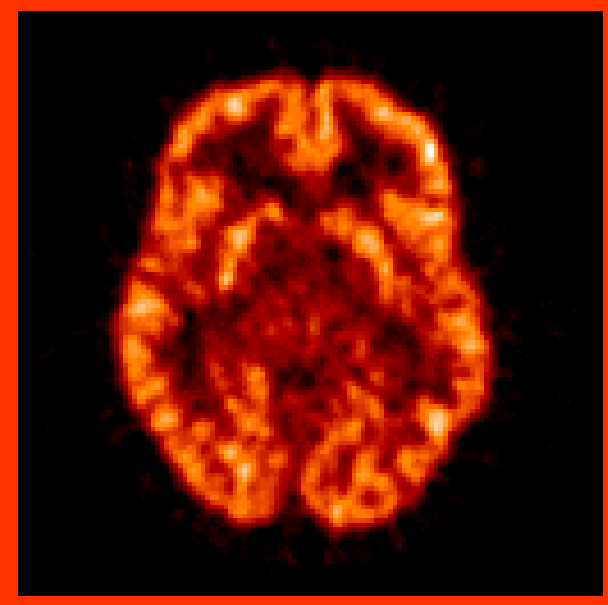

$2 \mathrm{D}$

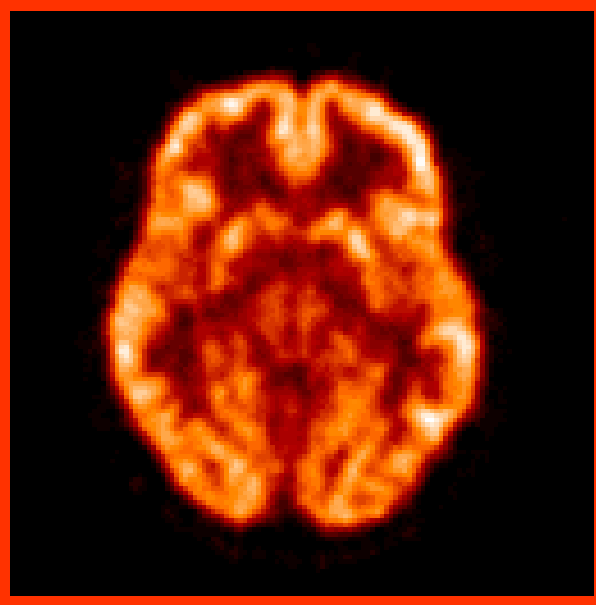

$3 \mathrm{D}$

- ROI analysis, particularly of hot-spots, yields different results for 2D and 3D modes.

- After perusing the existing phantom-oriented literature, we still were not sure which mode would be optimal for our FDG brain studies. 


\section{Goals:}

- Determine whether 2D or 3D mode is more appropriate for human $\left[{ }^{18} \mathrm{~F}\right]-\mathrm{FDG}$ brain scans on our GE/Advance PET scanner.

- Address concerns that 3D images are visually and perhaps quantitatively different than $2 \mathrm{D}$.

-Investigate possible tradeoffs in accuracy and reliability between 2D and 3D modes.

\section{Why should this be hard?}

- FDG brain scans typically have a similar NEC value for $2 \mathrm{D}$ and $3 \mathrm{D}$ modes.

- Phantoms tend to have simpler geometry (and hence simpler corrections) than humans.

- It is not straightforward how most phantom objects (point sources, uniform sources) translate to real-world image quality. 


\section{Human Subjects:}

Six (6) subjects were scanned on two occasions, in 2D and in 3D mode each time. The second occasion was 4-6 weeks after the first. Although these data were acquired as part of a different study, they are nearly ideally suited for a 2D/3D comparison. For the current work, each 2D/3D image pair was considered to be independent.

A 30 minute 2D emission scan was acquired starting at $~ 50$ minutes post-injection, followed by a 10 minute transmission scan, followed by a 10 minute $3 \mathrm{D}$ emission scan. The emission countrate fraction was $5 \%$ or less of the total countrate of the $\mathrm{T}+\mathrm{E}$ transmission measurement. Twice the total number of coincidence events were collected in 3D mode (100M) compared to 2D mode (40-50M); however, after scatter correction the total number of counts used for reconstruction was similar. A head-holding device was not used, but careful visual inspection revealed no detectable movement. Arterialized venous samples were collected, and the resulting measured input functions were used to calculate parametric images of $\mathrm{rCMR}_{\mathrm{glu}}$.

The image data were not spatially normalized to a common reference frame in this work, since we wanted to avoid any additional smoothing effects. Comparisons were made of each 2D/3D pair using the following metrics: image subtraction; histogram comparison; calculation of correlation coefficients (Pearson's) for pixels with values greater than a lower-level threshold of $1,80,90$, and $95 \%$ in either element of each $2 \mathrm{D} / 3 \mathrm{D}$ pair; and a regression analysis to investigate the effect of axial smoothing.

Phantom Data: A phantom with cold and hot spheres of various sizes in a warm background was scanned in $2 \mathrm{D}$ (Hi-Res) and 3D mode. The phantom's radioactive concentration $(0.5$ to $0.8 \mathrm{microCi} / \mathrm{ml})$ and volume $(2750 \mathrm{ml})$ were designed to simulate a $\left[{ }^{18} \mathrm{~F}\right]-\mathrm{FDG}$ PET scan of a human head. The phantom was a slightly tapered cylinder $(15.0 \mathrm{~cm}$ diameter in the center, $15.5 \mathrm{~cm}$ height) resting on one of its flat sides. This orientation was selected to reduce the possibility of overlapping artifacts from normalization (scanner dependent) and scatter (object dependent). The phantom contained 4 pairs of hollow plastic spheres (22, 17, 9, $4.7 \mathrm{~mm}$ ID) mounted on nylon thread; one sphere in each pair contained a nominal concentration of half of the warm background, and the other sphere in the pair contained twice the warm background. Four additional pairs of spheres (all $17 \mathrm{~mm}$ ID) positioned lateral to the variously sized spheres were used to obtain reliability estimates for ROI analysis. The two smallest "spheres" were in fact plastic cylinders with plugs in both ends, aligned so that the long axis of the cylinders were approximately vertical. The wall thickness of all of the plastic spheroids was 1.5 to $2.0 \mathrm{~mm}$.

Four scans of the phantom were acquired in 2D mode (30,15, 5, $1 \mathrm{~min})$, followed by four scans in 3D mode $(30,15,5,1 \mathrm{~min})$, followed by a 20 minute transmission scan. The standard software from the manufacturer was used for the normalization, scatter correction, attenuation correction (with a radioactive source present in the FOV), and reconstruction (2D: filtered backprojection, 30cm FOV, 128x128 pixels, 4.6 mm Hanning filter; 3D: Kinahan-Rogers filtered backprojection, $30 \mathrm{~cm}$ FOV, 128x128 pixels, transaxial 4.6mm Hanning filter, axial $8.5 \mathrm{~mm}$ Hanning filter). 


\section{Axial Resolution: by the numbers}

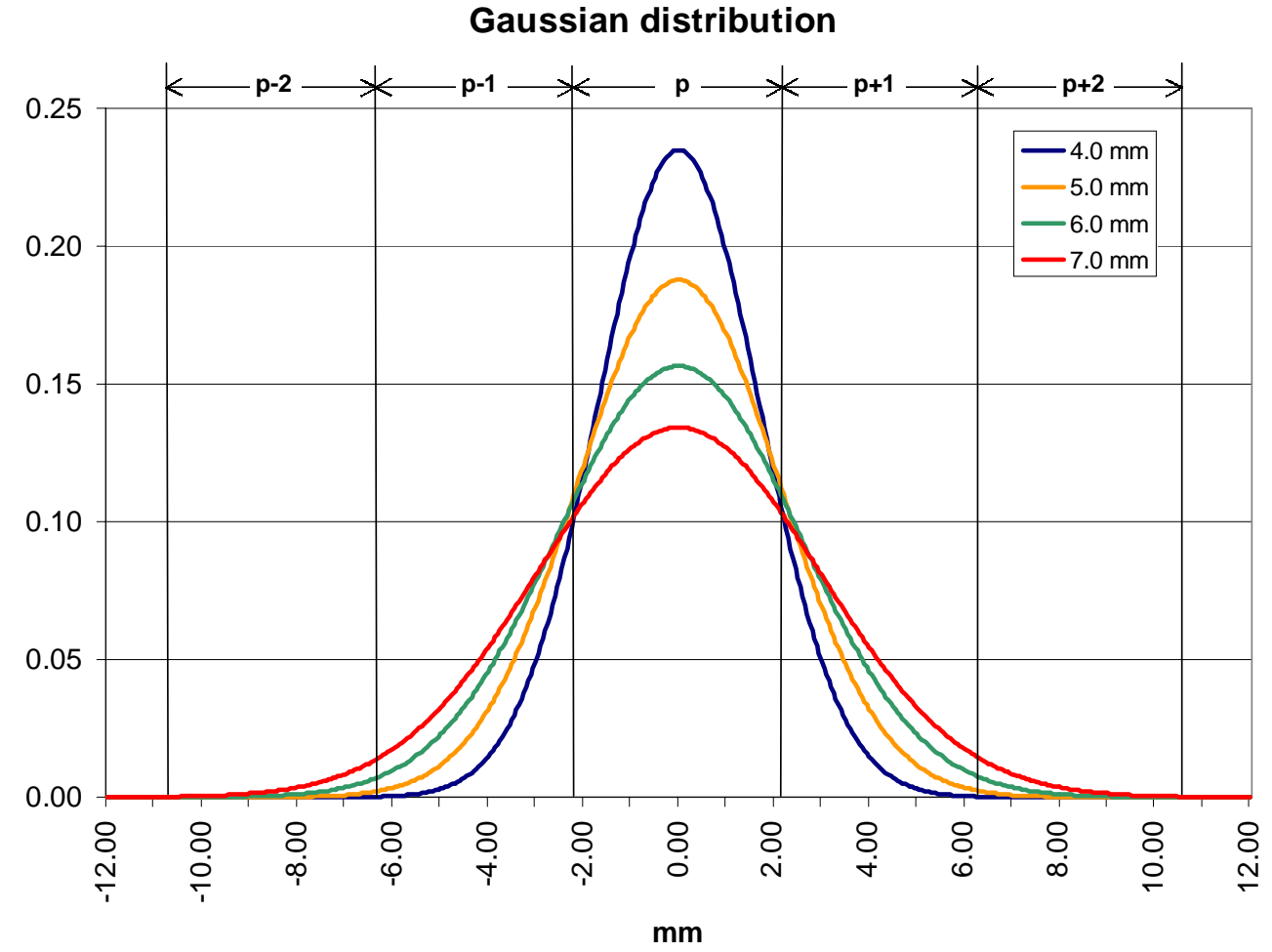

Fraction of events originating from a given plane and assigned to the current plane of interest
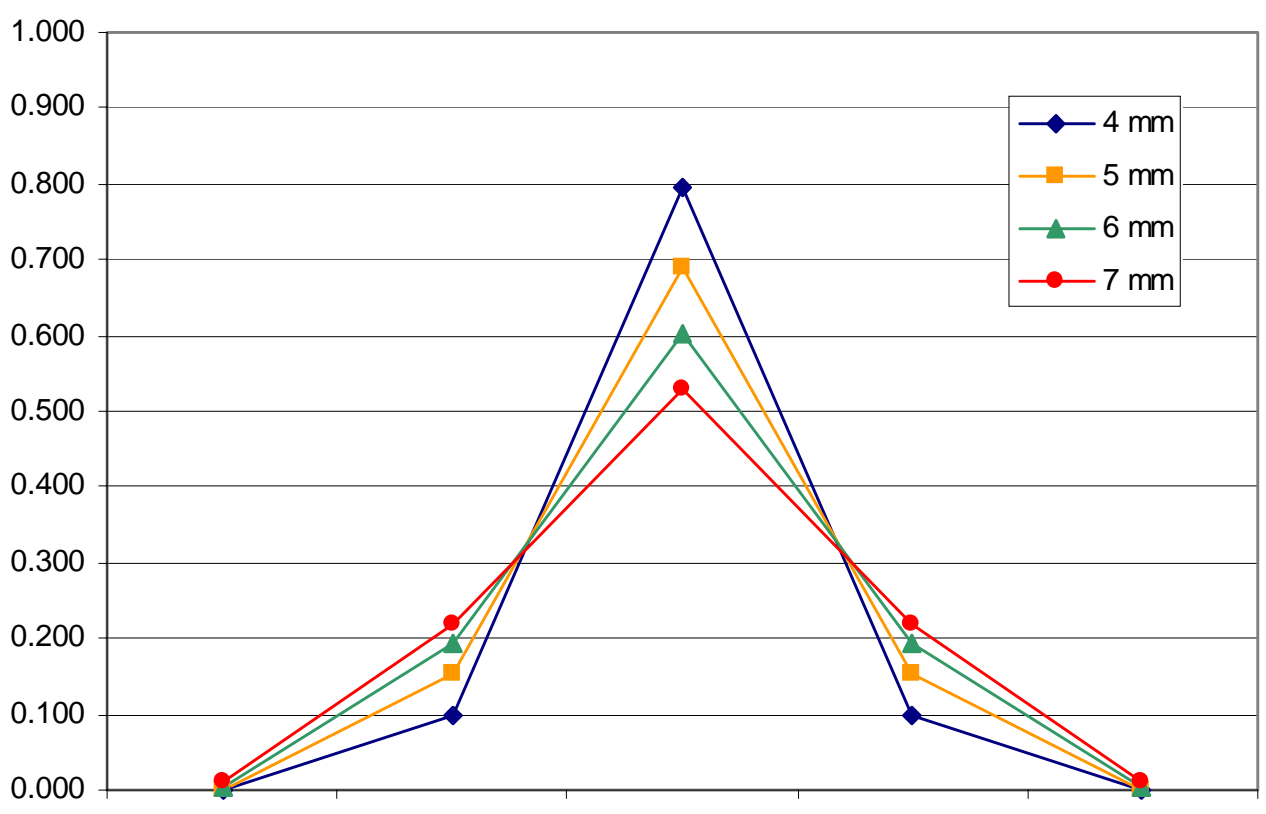

p-2 p-1 $p+1$

$\mathrm{p}+2$
Figure 1a.

Gaussian distributions with $\mathrm{FWHM}=4,5,6$, and $7 \mathrm{~mm}$. Vertical lines indicate separations between image planes for the GE/Advance (4.25mm).

Although the published differences in axial resolution appear small, even a difference in FWHM of $2 \mathrm{~mm}$ can have an effect on the fraction of counts from nearby locations contributed to a given image plane, and subsequently on axial image resolution. For an axial resolution described by a FWHM $=4.0 \mathrm{~mm}, 15 \%$ of the events recorded within a given plane originate from outside of the plane boundaries. Increasing the FWHM by only $2 \mathrm{~mm}$ means that $35 \%$ of the events are misplaced.

Figure 1b.

Fraction of events originating from one plane and assigned to the current plane, for axial resolutions of $\mathrm{FWHM}=4,5,6,7 \mathrm{~mm}$ and axial plane width $4.25 \mathrm{~mm}$ of GE/Advance PET scanner.

plane location relative to plane of interest 


\section{Axial Smoothing Filter: (can we make 2D look like 3D?)}

A location-dependent axial smoothing filter was constructed by culling values from published sources on the axial resolution of the GE/Advance in 2D mode (DeGrado 1994) and 3D mode (Lewellen 1996). A transaxial smoothing filter was not calculated, since this could make interpretation of results more difficult, and the transaxial resolutions only differ by $\sim 1 \mathrm{~mm}$ or less. For radii from $0-20 \mathrm{~cm}$, and for each image plane, the reconstructed axial resolution was determined using published values, interpolating linearly between values where necessary. The fraction of a Gaussian distribution in an image plane was calculated for the current plane of interest (p0) and the nearest 4 planes ( $\mathrm{p}-2, \mathrm{p}-1, \mathrm{p}+1, \mathrm{p}+2)$, for Gaussian distributions centered on $\mathrm{p} 0$ with FWHM for 2D and $3 \mathrm{D}$ modes at each voxel in an image volume. The fractions are named as $\mathrm{F}_{2} \mathrm{D}_{\mathrm{p}}$ and $\mathrm{F}_{\mathrm{p}} \mathrm{D}_{\mathrm{p}}$, where $\mathrm{p}$ is the number of planes from the current plane. Weighting factors for nearby pixels in the axial dimension were calculated as:

$$
\mathrm{w}_{\mathrm{p}}=\mathrm{F} 3 \mathrm{D}_{\mathrm{p}} *\left(1-\mathrm{F} 2 \mathrm{D}_{\mathrm{p}}\right)
$$

which yields the fraction of the 3D contribution from a given plane not already accounted for by the 2D contribution. In the plot below, this is represented by the area with the red hash pattern.

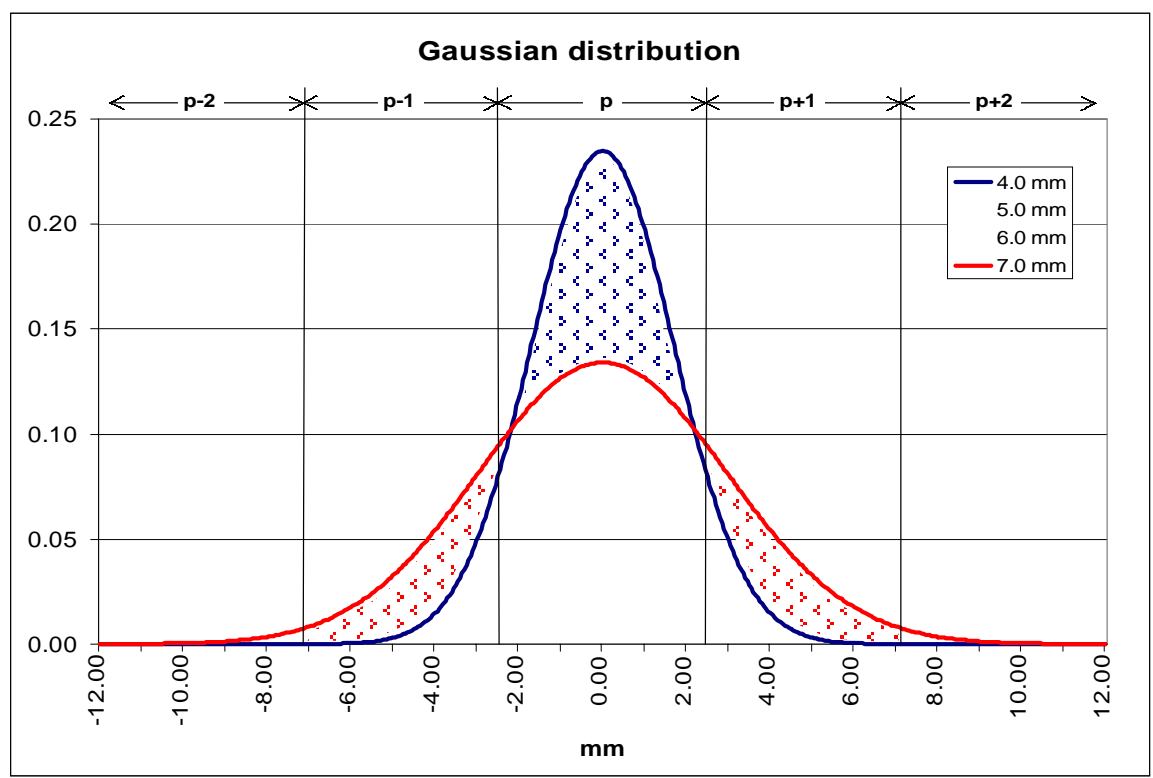

Figure 2. Different resolutions yield different fractions of mis-assigned events.

To apply the smoothing filter to the 2D images, each pixel was asigned the weighted average of itself and the corresponding pixels from the neighboring +/-2 planes:

$$
\mathrm{C}(\mathrm{x}, \mathrm{y}, \mathrm{p})=\mathrm{C}_{\mathrm{p}}=\underset{\mathrm{i}=\mathrm{p}-2}{\mathrm{p}+2} \mathrm{w}_{\mathrm{i}} \mathrm{C}_{\mathrm{i}}
$$

If the Gaussian distribution extended beyond $\mathrm{p}+/-2$, the remainder was evenly shared with $\mathrm{p}+/-1$, $\mathrm{p}+/-2$. For planes near the end of the FOV, weights for planes which would be outside the axial FOV were evenly shared by $\mathrm{p} 0$ and $\mathrm{p}-1$ or $\mathrm{p}+1$, if applicable. Weighting factors for $\mathrm{p}-2, \mathrm{p}-1, \mathrm{p} 0, \mathrm{p}+1, \mathrm{p}+2$ were then multiplied by the values of the single pixel from each corresponding plane with the same $\mathrm{x}, \mathrm{y}$ location as the current pixel of interest. This approach differs from an axial smoothing filter presented by Pajevic et al. (1998) in that it yields axially- and radially-dependent smoothing kernels. 


\section{Figure 3.}

The 3D images appear markedly smoother, even though there is a similar number of total counts in each of the corrected sinogram sets.
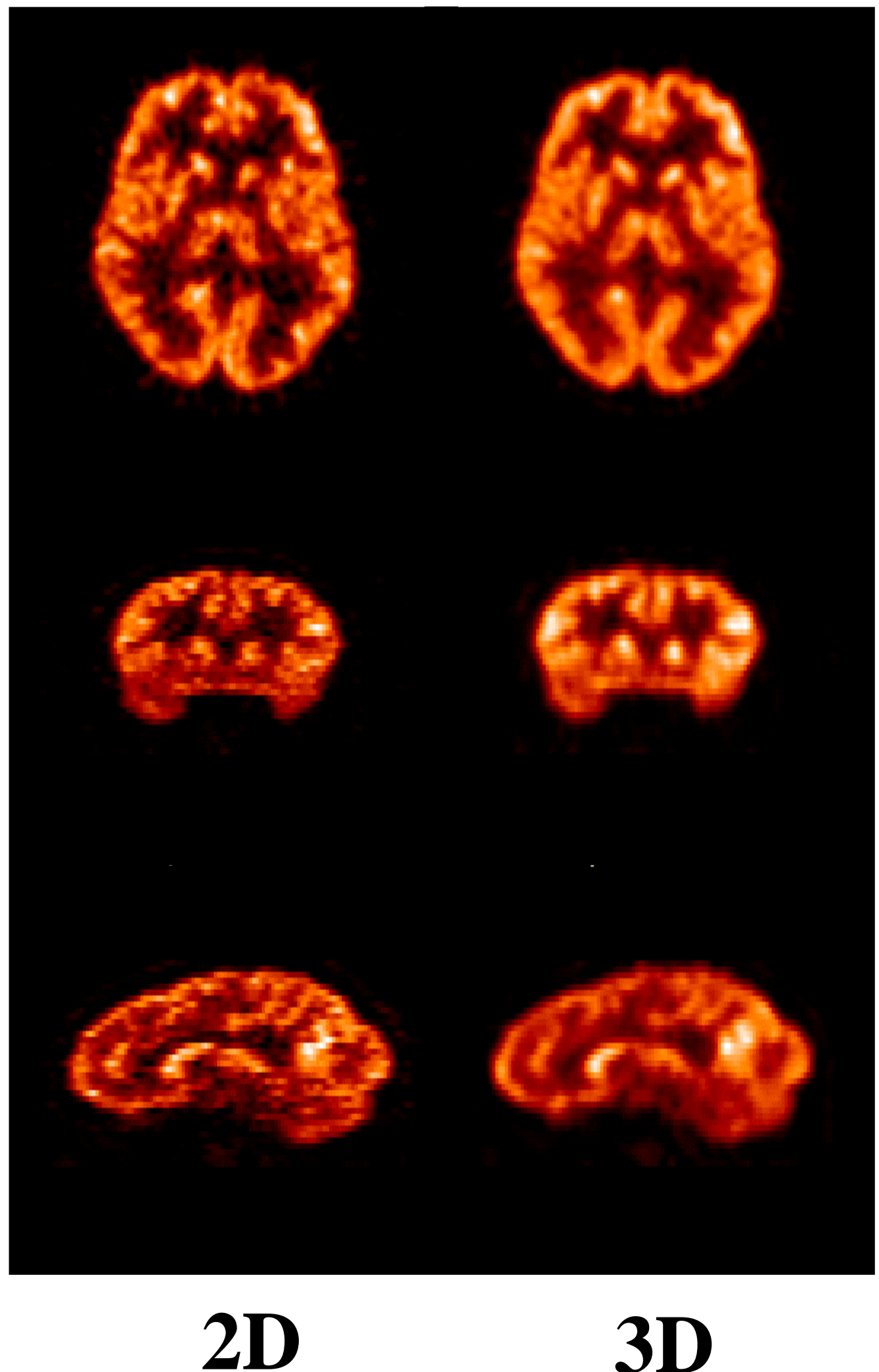

3D 


\section{Results}

Figure 4. The difference in average metabolic rate between the individual 2D and $3 \mathrm{D}$ pairs ranges from $0.0 \%$ to $4 \%$. This holds true if all pixels are included in the average, or if pixels with values below 0.1 are excluded from the average.

\section{Average metabolic value}

(for pixels greater than 0.1 from any image volume)

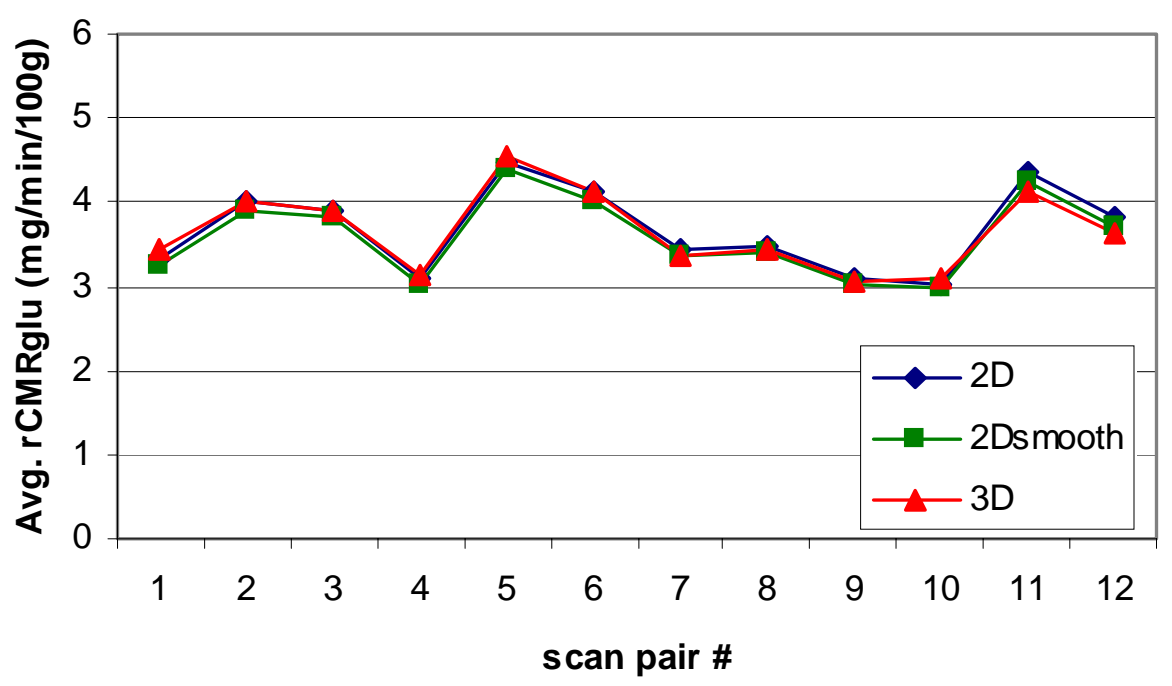

Figure 5. Image subtraction yields a consistent pattern of greater values for hot-spots and lower values for cold-spots in the 2D images, as demonstrated below. The numbers above the images show the value at the crosshair location. This effect is consistent throughout the brain volume, for all subjects.

13.08

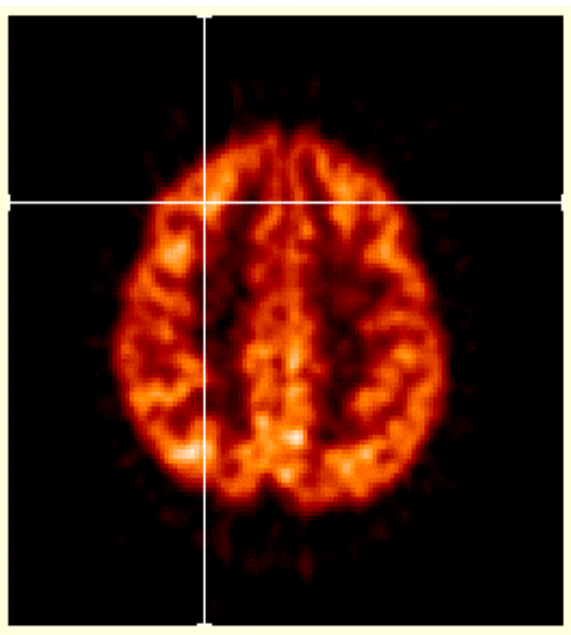

9.80

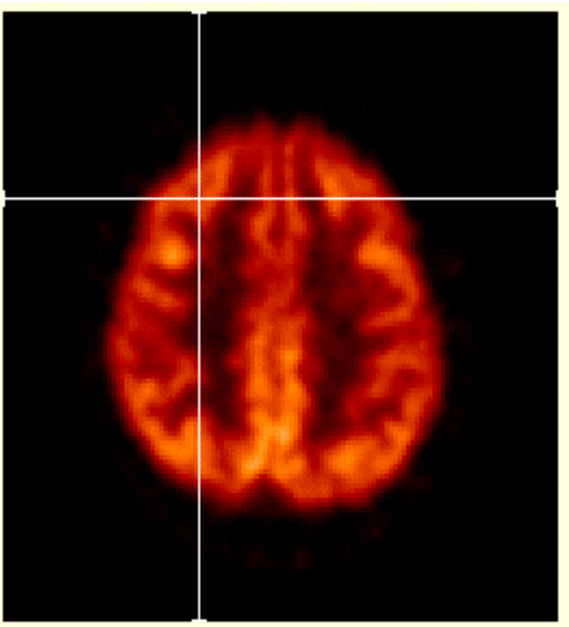

\subsection{9}

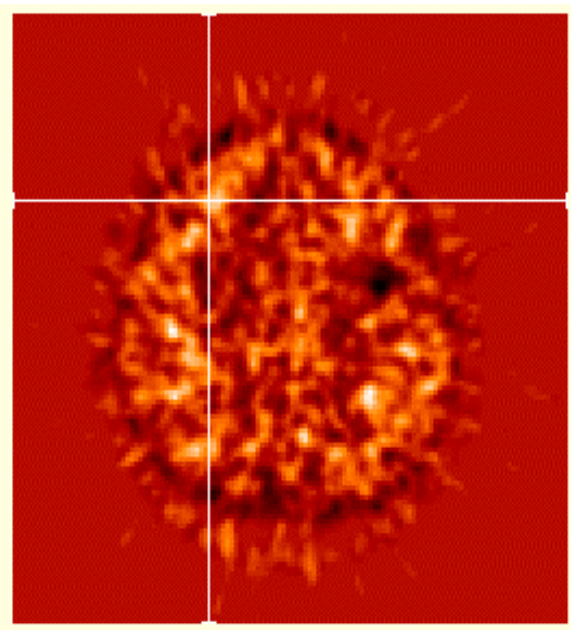




\section{Results: Image subtraction}

Figure 6.

There is a distinct "halo" in the subtraction images near the edge of the brain.This is particularly prominent near the top of the brain. This could be due to differences in scatter-correction between 2D and 3D modes, or to the effect of axial smoothing which makes the apparent size of the brain larger in regions where the brain shape changes rapidly from one axial plane to the next. Comparison of 2D axially smoothed images with $3 \mathrm{D}$ images shows a similar pattern but with a reduced magnitude.
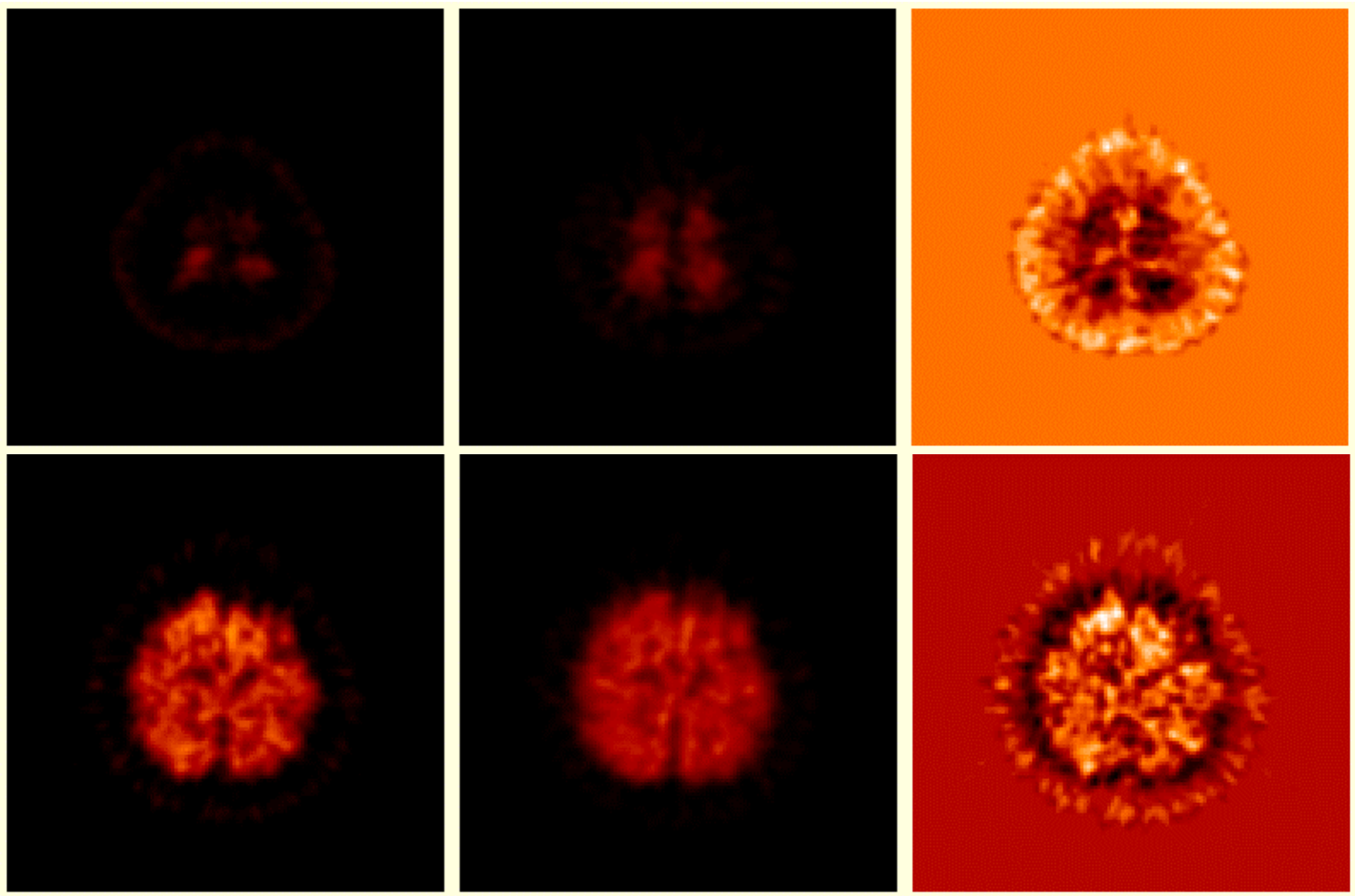

plane 34
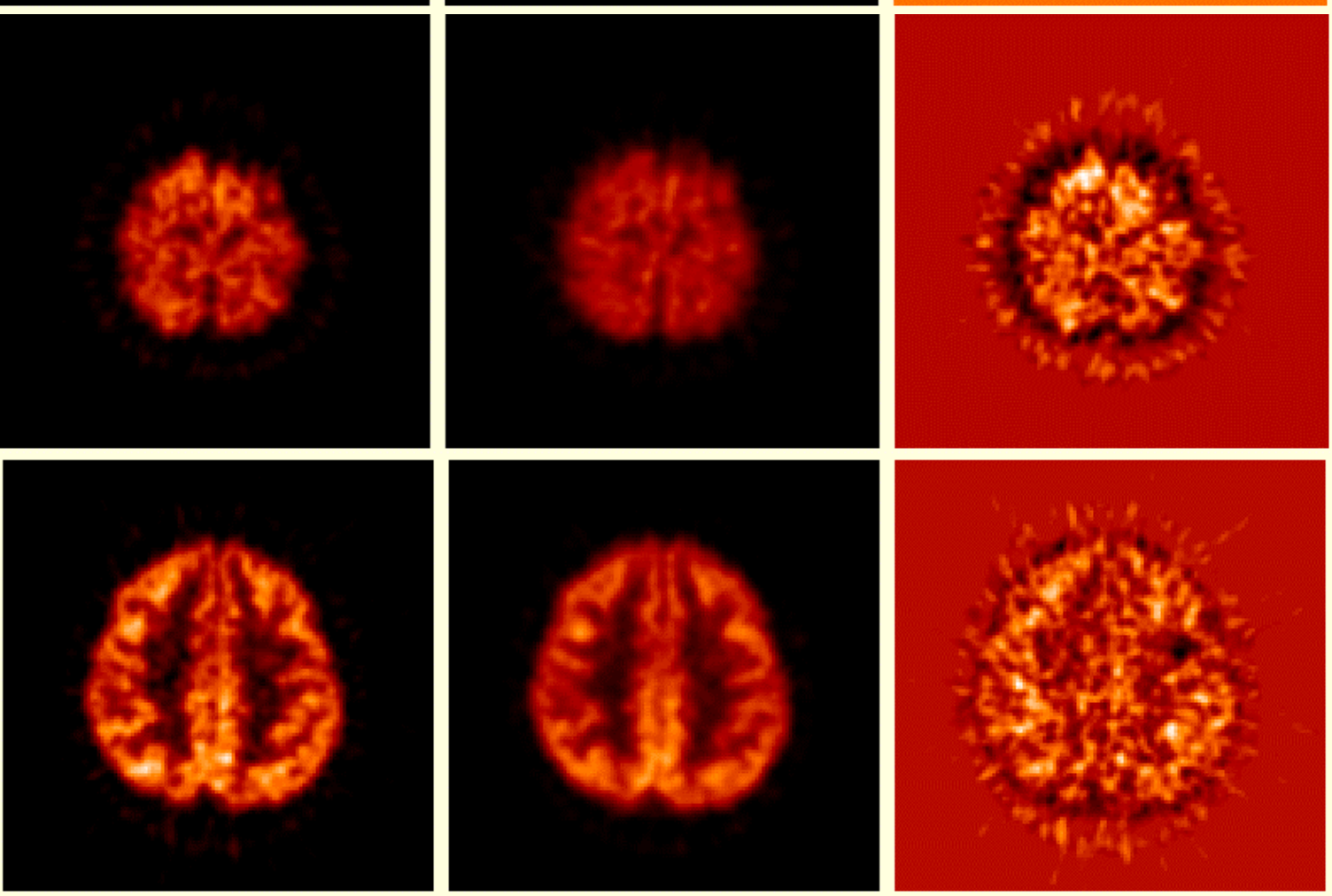

plane 31

plane 26

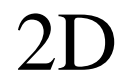




\section{Results: Histogram Analysis}

\section{Figure 7.}

Comparing histograms from individual 2D/3D pairs shows that the largest and smallest values from the 2D images become pushed toward the average value in the $3 \mathrm{D}$ data; regions which are most or least active in the 2D images become relatively less or more active in the 3D data. This can be an important difference for analysis involving ROI placement on hotspots or searching for areas of maximal activation, since these are the pixels that differ most between $2 \mathrm{D}$ and 3D.

For all pixels with a value above 0.1 (in either element of a 2D/3D pair), the fraction of the number of values in the 2D histograms larger than the corresponding 3D histogram values has a mean of 0.113 for all 12 pairs; comparing the axially smoothed 2D data to the $3 \mathrm{D}$ data yields a ratio of 0.071 .

The histograms shown below are from a single representative subject. The green lines indicate the thresholds for percentiles of 85, 90, and 95. Note the log-based ordinate.

\section{Histograms of a paired 2D, 3D image volume}

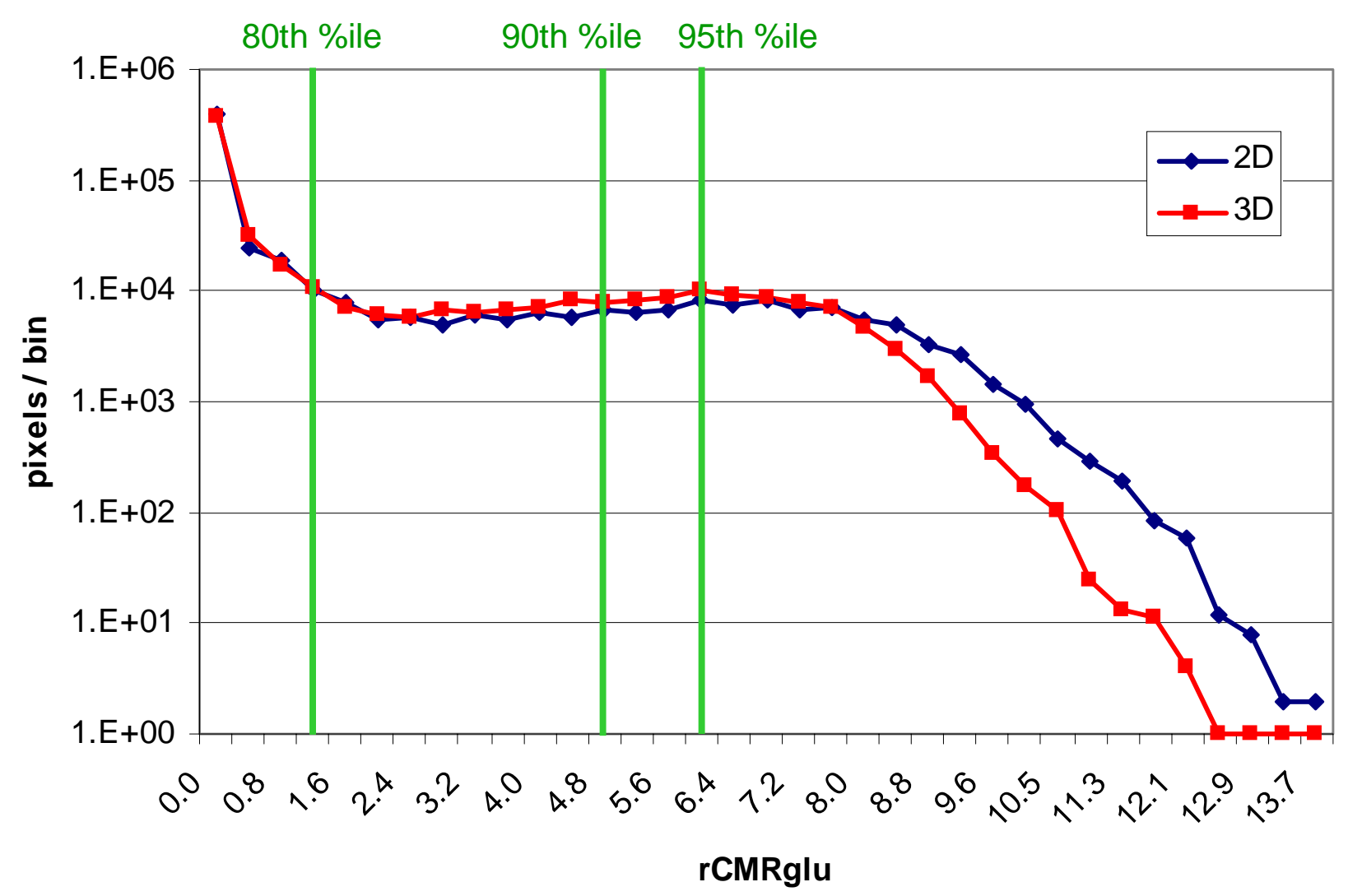



(2D-smoothed, 3D) FDG image volumes

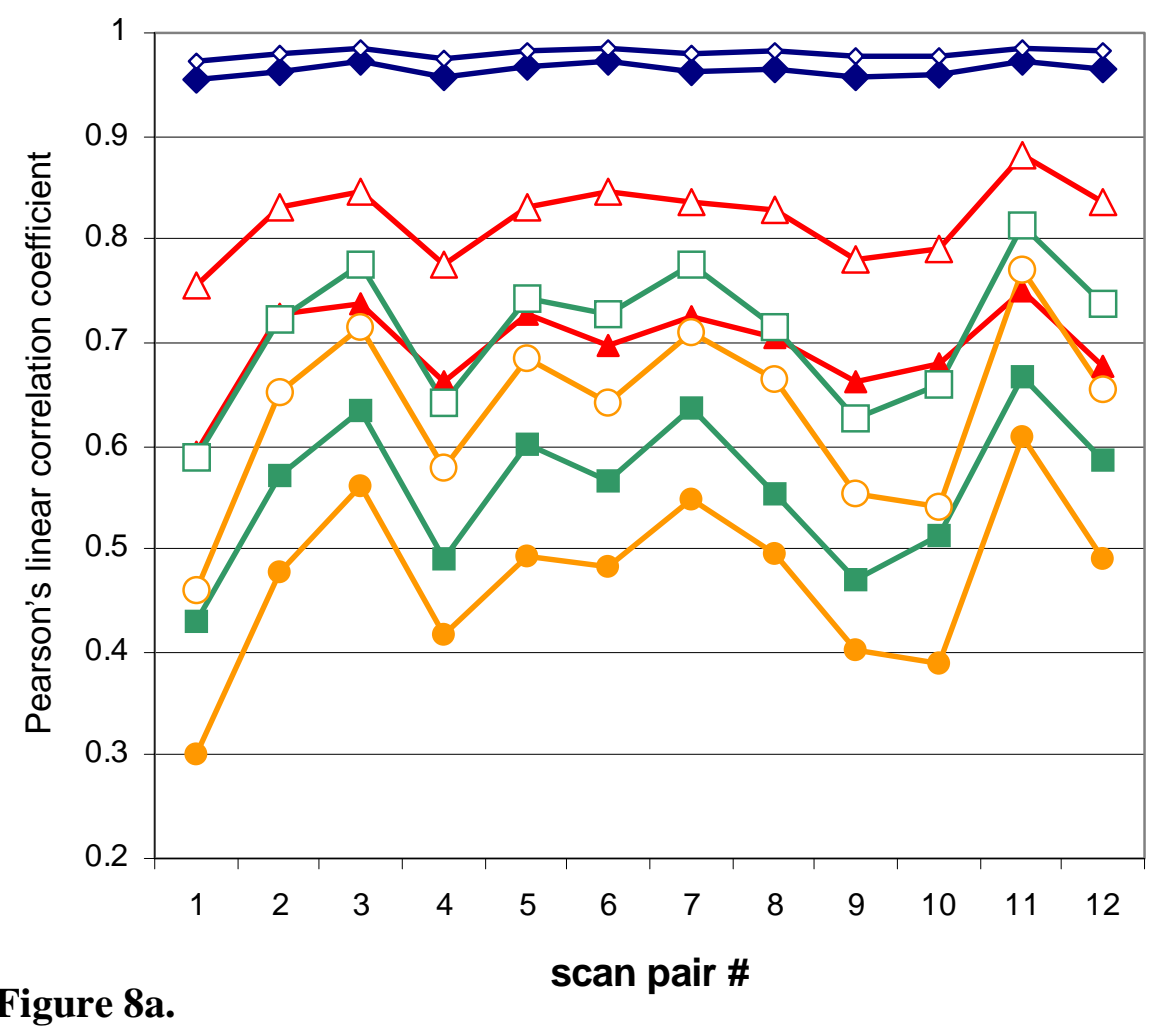

$\neg 1 \%$ ile: 2D, 3D

$\neg 1 \%$ ile: 2Dsmooth, 3D

$\rightarrow-80 \%$ ile: 2D, 3D

$\triangle-80 \%$ ile: 2Dsmooth, 3D

$-90 \%$ ile: 2D, 3D

$-\square-90 \%$ ile: 2Dsmooth, 3D

-95\%ile: 2D, 3D

$-0-95 \%$ ile: 2Dsmooth, 3D

The correlation analysis shows that overall (for all values above the minimum threshold of 0.1 ) the 2D and $3 \mathrm{D}$ image sets are well correlated (see Fig. 8a, plots for $1 \%$ ile).

As the minimum pixel value considered is raised, the correlation coefficient decreases. One possible interpretation is that the noise component increases for groups of pixels with higher values, so other factors become less important. However, taking into consideration the histogram analysis, it is likely that a there is a larger discrepency between $2 \mathrm{D}$ and $3 \mathrm{D}$ mode as the pixel value increases.

Axially smoothing the 2D data increases the correlation, indicating that the smoothed 2D data are more similar to the 3D data.

Figure 8b. 


\section{Distance of bin Center-of-Mass from $y=x$ (average for 12 scan pairs)}

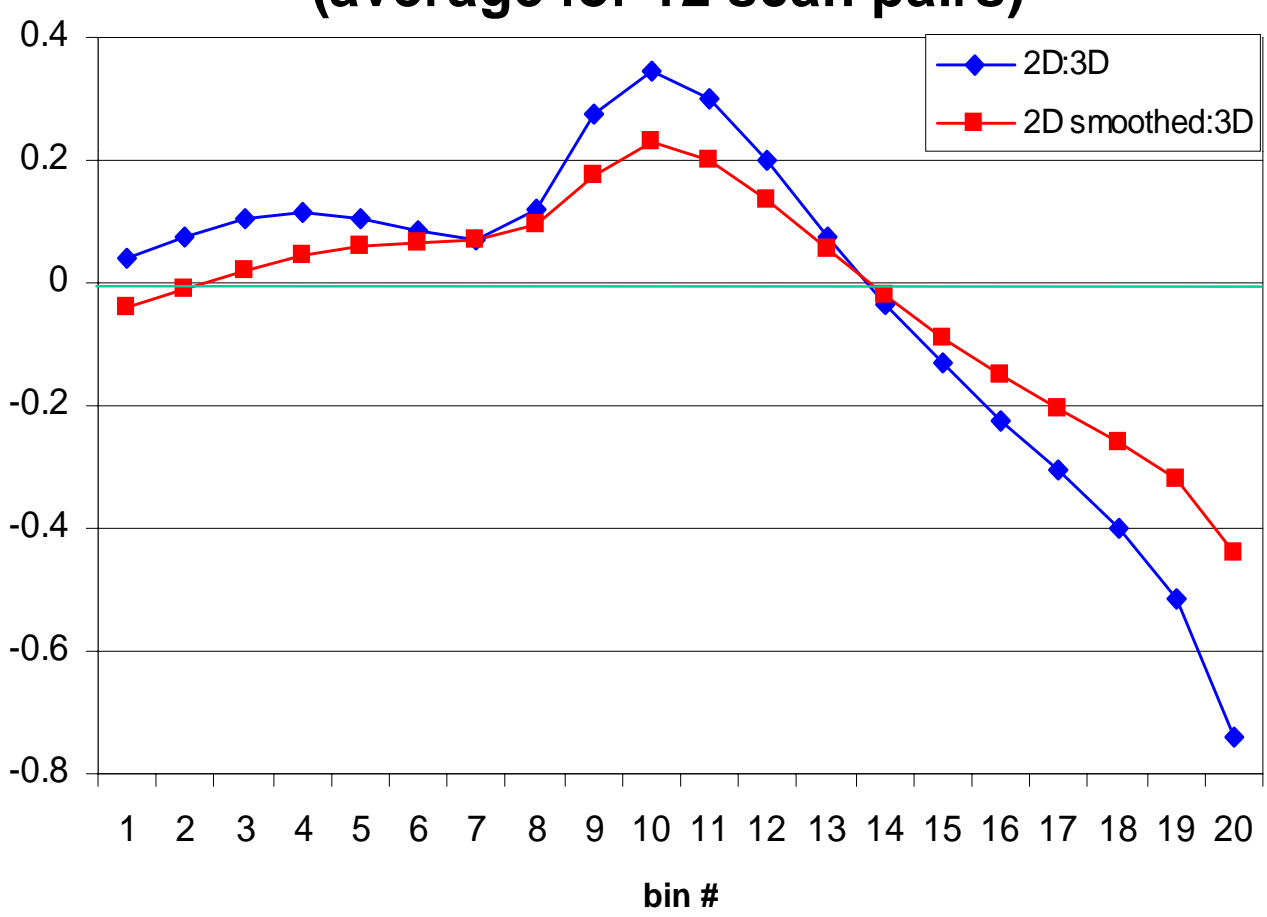

Figure 10a.
Figure 10a. The distance of the Center-of-Mass (CoM) in each bin was averaged over all 12 FDG scans for 2D:3D and 2D-

smoothed:3D. In all bins except the first, the CoM in the axially smoothed pair is smaller, indicating a better match with the 3D data.

More interesting is the pattern of the 2D:3D plot. Positive values indicate bins where the 3D data tend to be higher than the 2D data, and negative values show where the 3D data are lower. Areas of higher concentration (hotspots) have reduced values compared to the 2D data, while cold-spots have increased values.

\section{Fraction of difference between 2D, 3D scans attributed to 3D axial smoothing}

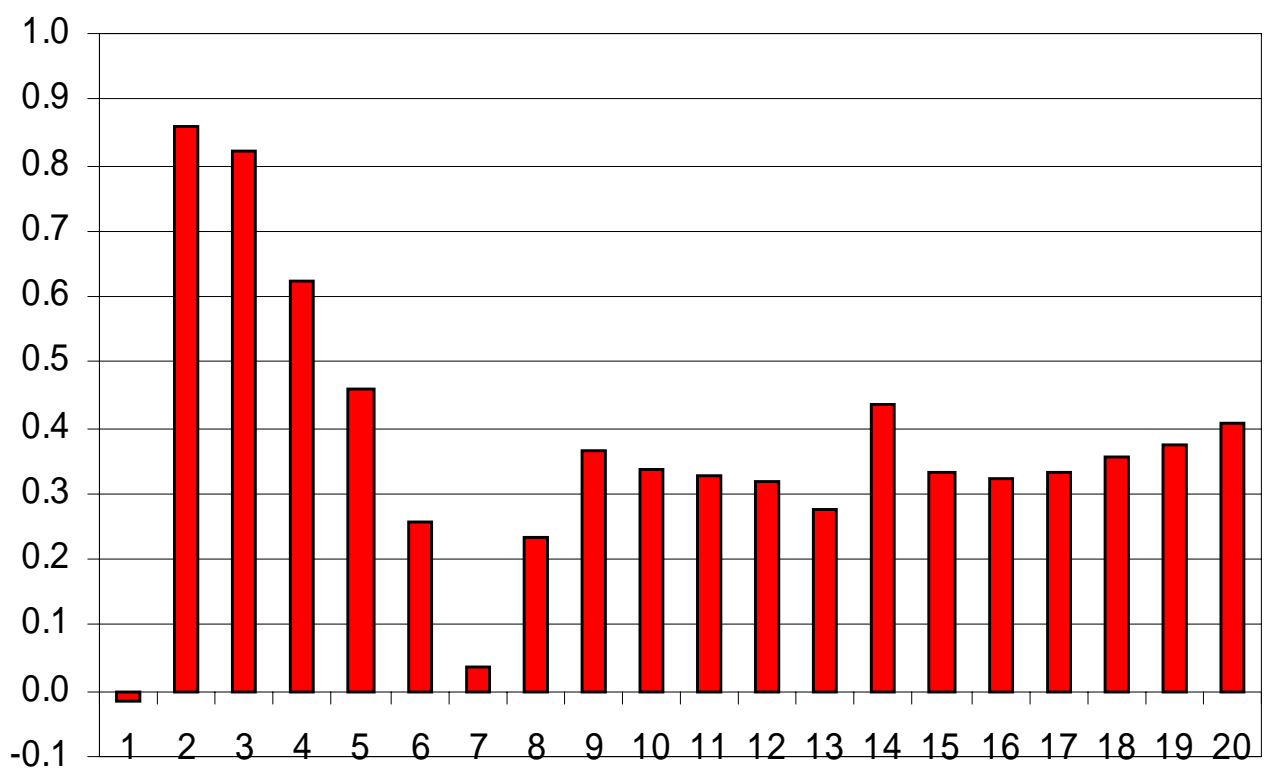

Figure 10b. bin \#
Figure 10b. The fraction of the difference between images acquired in $2 \mathrm{D}$ and $3 \mathrm{D}$ mode that can be explained by the axial smoothing filter is shown for each bin, calculated as:

$$
\mathrm{f}=1-\left(\frac{\mathrm{d}_{\mathrm{CoM}}(2 \mathrm{D} \text { smoothed })}{\mathrm{d}_{\mathrm{CoM}}(2 \mathrm{D})}\right)
$$

Differences between 2D and $3 \mathrm{D}$ modes due to 3D axial smoothing are fairly constant throughout the bins with medium to higher levels of concentration, and can account for $30 \%$ to $40 \%$ of the observed differences between these two modes. 


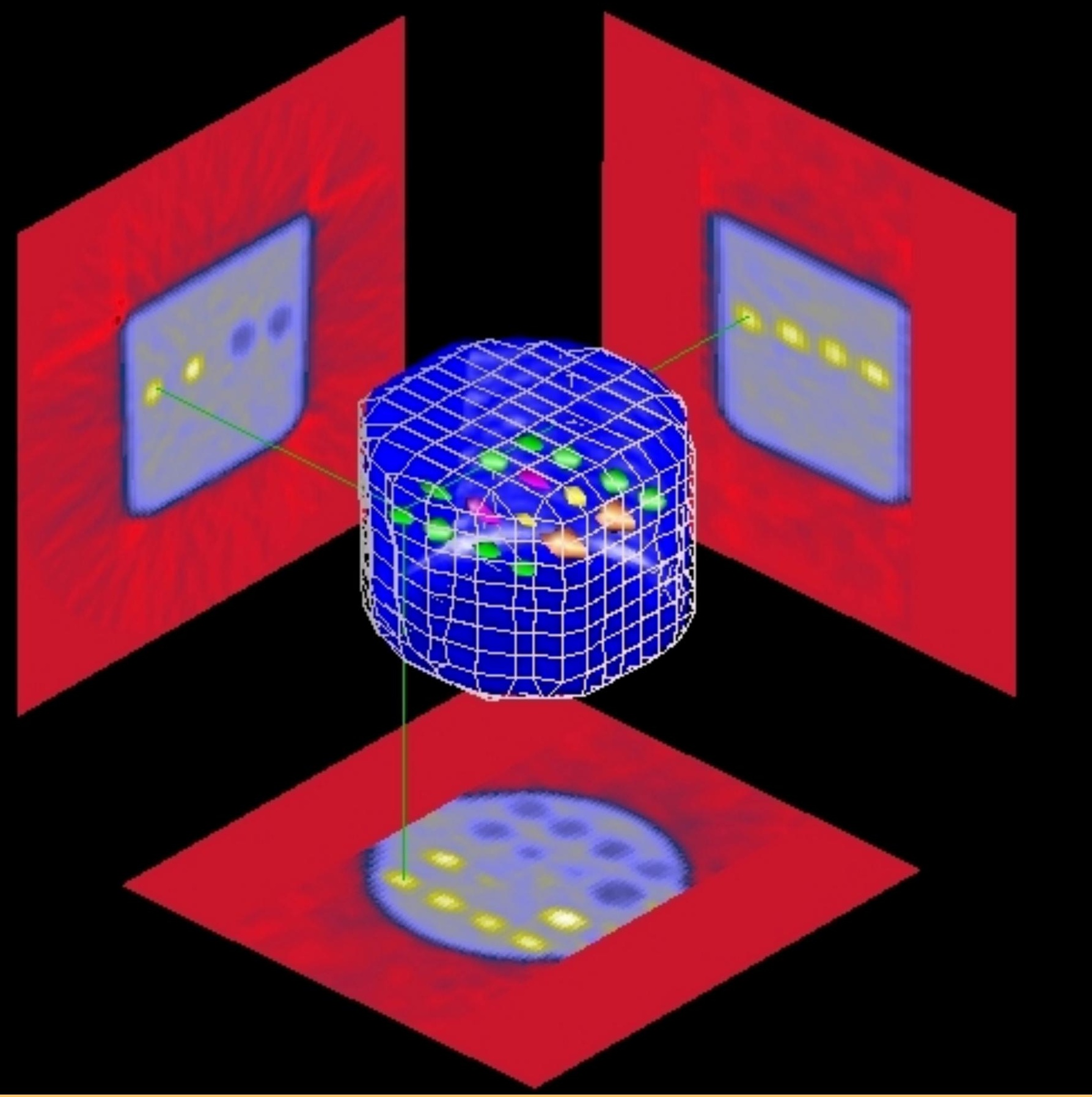

Figure 11. Rendered view of the shere-filled phantom with a warm background. Three orthogonal planes are shown, corresponding to axial (back left), coronal (bottom) and sagittal (back right) for a supine human head. The phantom contained a warm background $(0.8 \mathrm{mCi} . \mathrm{ml}$ for the $2 \mathrm{D}$ scan, $0.5 \mathrm{mCi} / \mathrm{ml}$ for the $3 \mathrm{D}$ scan) with a total volume of $2750 \mathrm{ml}$.

The rendered view in the center shows the shape of the phantom with the various spheres in the center (orange $=22 \mathrm{~mm}$, green $=17 \mathrm{~mm}$, purple $=9 \mathrm{~mm}$, yellow $=4.7 \mathrm{~mm}$ ). The spheres on the left side contained twice the concentration of the background, and the spheres on the right contained half of the background concentration. 


\section{Results: Spheroid Recovery Coefficients.}

Goal 1 for this work could be rephrased as, "Given that we frequently draw ROIs on our FDG PET data for objects like the amygdala, which mode should we use?"

The graph in Figure 12 shows the recovery coefficients we obtained for hot and cool spheres relative to a warm background. The results for the hot spheres are similar to those obtained previously (Lewellen, 1996) for a broader range of diameters. Here, there is better agreement between the two modes for the cool spheres than the hot spheres, particularly for the two smaller diameters. We attribute this mainly to the thick walls of the spheroid containers used for these two sizes, which introduced a significant cold region next to the warm or cool sphere interior.

The average values for 5 hot and 5 cold $17 \mathrm{~mm}$ diameter spheres are shown in Figure $13 \mathrm{a}$, for 4 different time frames. The concentration for each 3D frame was $68 \%$ of the corresponding $2 \mathrm{D}$ frame. The $3 \mathrm{D}$ averages are similar to the $2 \mathrm{D}$ averages, but reflect the differences in recovery coefficients shown in Figure 12.

The standard deviation of these values is shown as error bars in Figure 13a, and plotted on a larger scale in Figure 13b. We use this as our estimate of the reliability for ROIs drawn in each mode. The standard deviation is similar for both modes except for the shortest frame (1 minute) where $3 \mathrm{D}$ mode shows an advantage.

\section{Recovery Coefficients for Hot and Cold spheres}

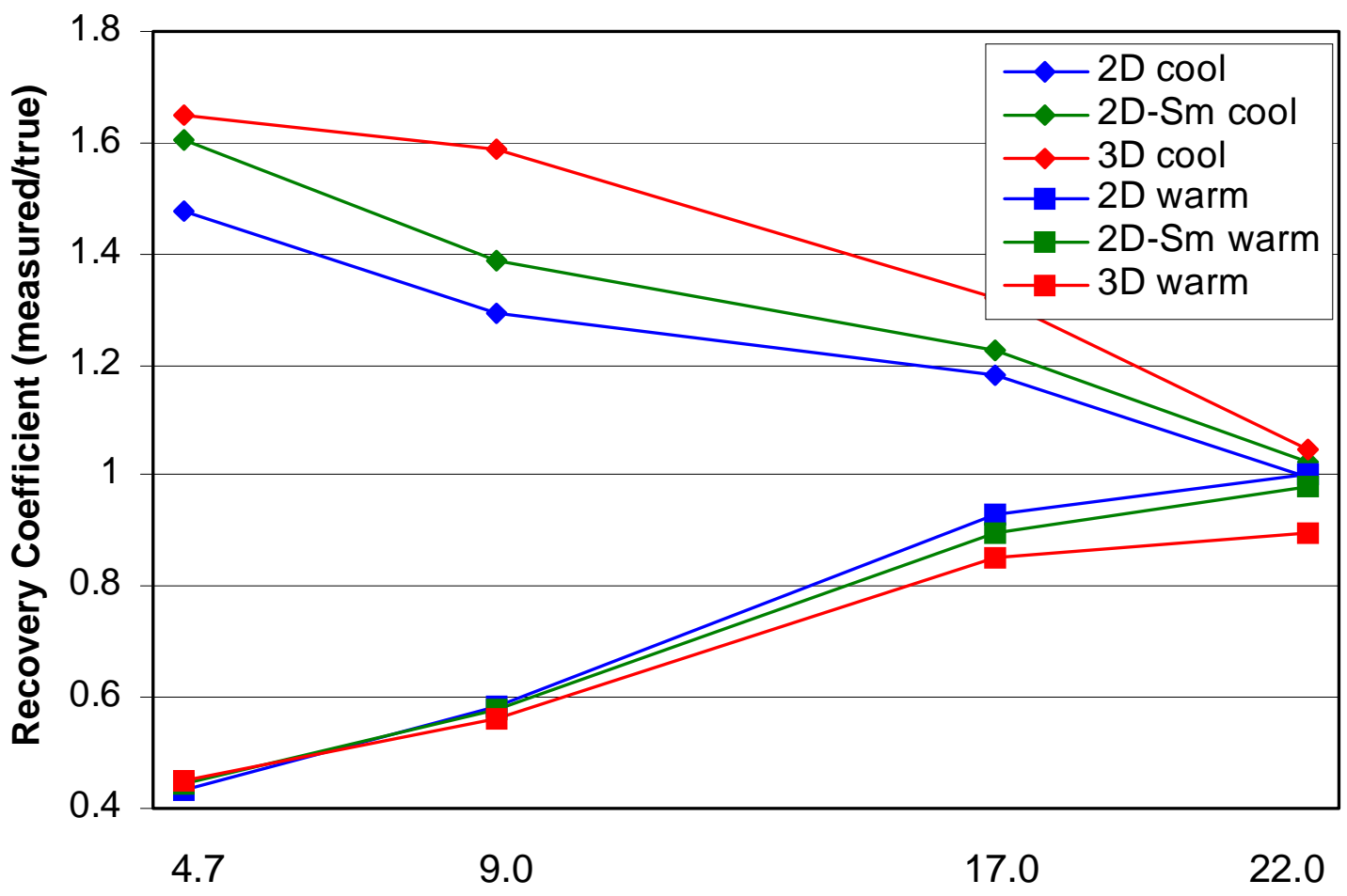

Figure 12.

Sphere Diameter $(\mathrm{mm})$ 
ROI average values for $17 \mathrm{~mm}$ spheres

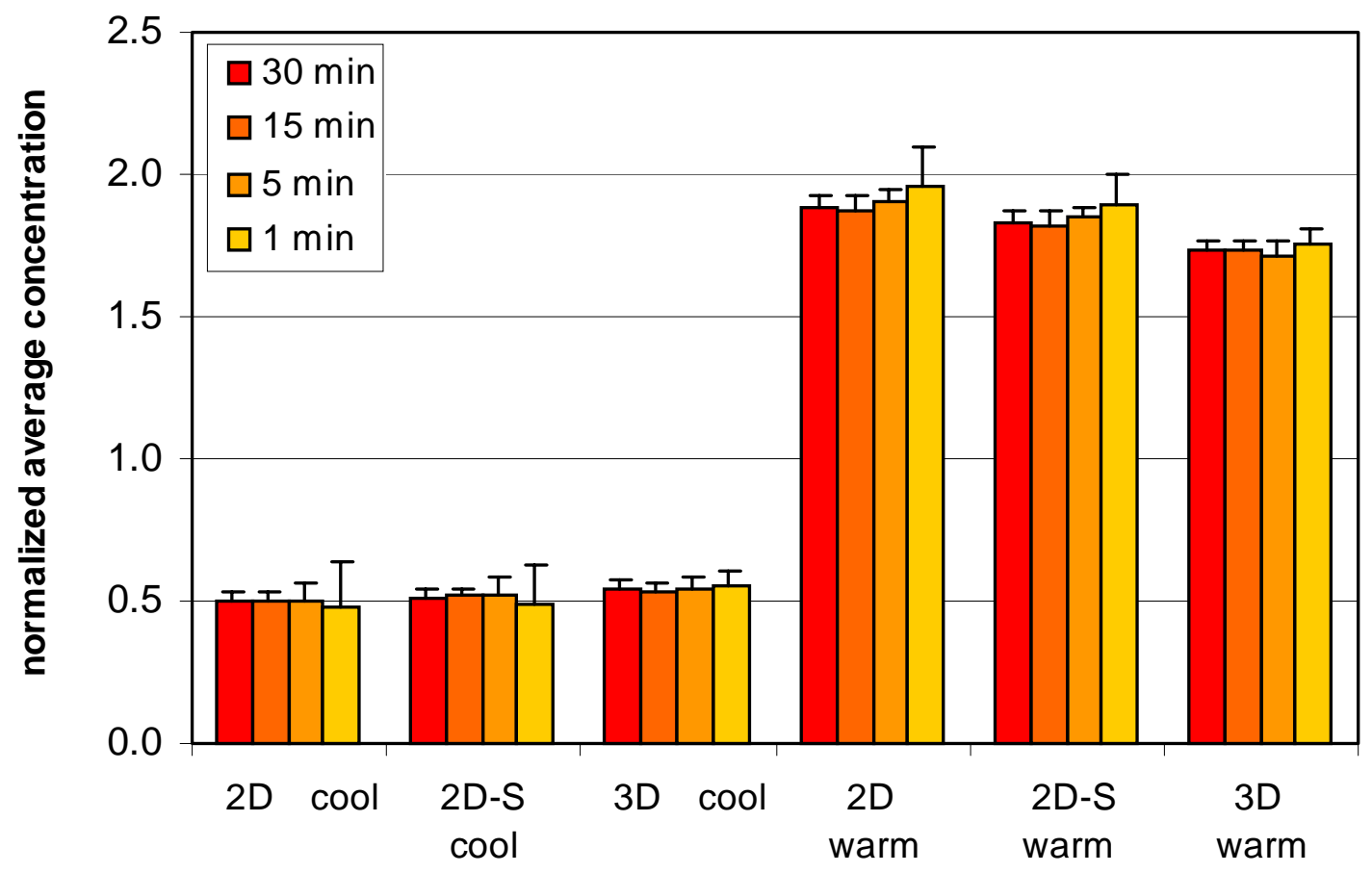

Figure 13a.

Standard deviation of ROI values for various acquisition durations

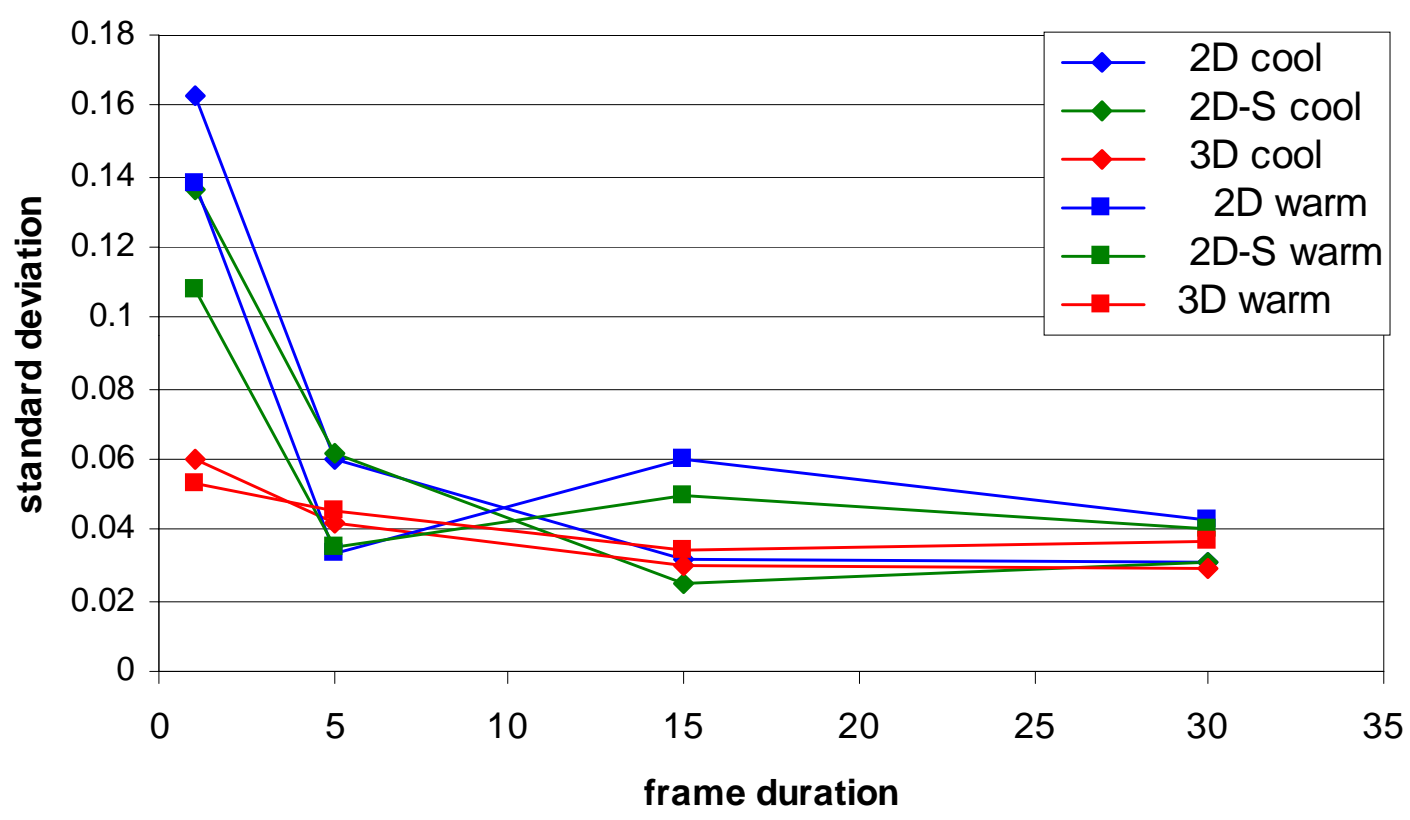

Figure 13b. 


\section{Conclusions:}

- Only applicable for GE/Advance PET scanner.

- For a typical human-subject cerebral FDG PET scan, slightly greater quantitative accuracy is obtained with 2D mode instead of 3D mode.

-Differences between 2D and 3D modes are greatest for large and small values, which can adversely affect certain types of image analysis.

- The larger number of counts obtained in 3D mode has little effect on the reliability of ROI values obtained for a $17 \mathrm{~mm}$ sperical ROI for typical FDG brain scan conditions.

- 3D mode shows an advantage in ROI reliability for data with an unusually low number of decay-event counts.

\section{References:}

DeGrado TR, Turkington TG, Williams JJ, Stearns CW, Hoffman JM, Coleman RE, "Performance characteristics of a whole-body PET scanner", J. Nucl. Med., 35:1398-1406, 1994.

Hochachka PW, Clark CM, Brown WD, Stone CS, Nickles RJ, Holden JE, "Effects on regional brain metabolism of prolonged high altitude exposure. A study of six U.S. Marines", Am. J. Physiol., 46:R314- 319, 1999.

Kinahan RE, Rogers JG, "Analytic 3-D image reconstruction using all detected events", IEEE Trans. Nucl. Sci., 36(1):964-968, 1989.

Lewellen T, Kohlmyer S, Miyaoka R, Schubert S, Stearns C, "Investigation of the count rate performance of the General Electric ADVANCE positron emission tomograph", IEEE Trans. Nucl. Sci., 42:1051-1057, 1995.

Pajevic S, Daube-Witherspoon ME, Bacharach SL, Carson RE, "Noise characteristics of 3-D and 2-D PET images", IEEE Trans. Med. Imag., 17(1):9-23, 1998. 